\title{
ESTUDIOS
}

\section{Vestigios de una lengua originaria en el territorio de la cultura chachapoya}

\author{
Vestiges of a native language in the territory \\ of the Chachapoyas culture \\ JAIRO VALQUI \\ Universidad Nacional Mayor de San Marcos \\ jvalquic@unmsm.edu.pe \\ MiCHAELA ZIEMENDORFF
Universidad de Colonia
mziemendorff@gmail.com
}



Resumen

Previas investigaciones basadas principalmente en la toponimia y antroponimia de la zona antiguamente habitada por los pueblos de la cultura chachapoya en el sur del departamento de Amazonas han permitido establecer la hipótesis de una lengua originaria preincaica, sustituida por el quechua antes de la Conquista. Las fuentes disponibles, sin embargo, no dan ninguna razón de dicha lengua, por lo que el presente artículo recurre a un análisis linguíístico para determinar si la toponimia y antroponimia han conservado vestigios de una o varias lenguas en la región ocupada por esta cultura y si, por lo tanto, es justificado referirse a una «lengua chacha». Este estudio desarrolla principalmente un trabajo de campo enfocado en el análisis de vestigios toponímicos prequechuas que permite sostener vestigios de una lengua originaria en parte del territorio de los chachapoya. 
Palabras claves: toponimia, antroponimia, cultura chachapoya, «lengua chacha», Amazonas

\begin{abstract}
Previous research which focused on the toponymy and anthroponymy of the ancient peoples of the Chachapoyas culture in the southern portion of the Amazonas region has led to the hypothesis of a pre-Inca original language, later supplanted by Quechua prior to the Spanish Conquest. The extant historical documents, however, do not mention any such pre-Quechua language in this geographical area. This article presents a linguistic analysis to determine if the toponymy and anthroponymy have preserved vestiges of one or more languages in the region occupied by this culture, and therefore, if it is justifiable to refer to a chacha language. The study concerns itself primarily with field work focused on the analysis of pre-Quechua place names that sustain the concept of an original language in part of the Chachapoyan territory.
\end{abstract}

Keywords: Toponymy, Anthroponymy, Chachapoyas culture, Chacha language, Amazonas.

Recibido: 15/4/16 Aceptado: 20/5/16

\title{
Introducción
}

Los documentos coloniales más importantes que han sido encontrados hasta el momento sobre el pasado de las provincias de Chachapoyas y Luya en el sur del departamento de Amazonas han reportado en términos linguísticos la existencia de una sola lengua anterior al castellano: el quechua. Documentos como el primer libro de Cabildos de San Juan de la Frontera de los Chachapoyas de 1538 o el expediente del Repartimiento de Leymebamba y Cochabamba (1572-74) atestiguan que los pobladores locales utilizaban la lengua del inca ${ }^{1}$. Sin embargo, los topónimos y antropónimos consignados en los archivos locales y de uso actual evidencian la posible existencia de una o más lenguas distintas al castellano y al quechua en esta parte del departamento de Amazonas.

Estos indicios y otros, analizados en su momento por estudiosos como Langlois (1939), Zevallos Quiñones (1966), Torero (1989) y Taylor (1990), han

1 En el expediente Repartimiento de Leymebamba y Cochabamba se atestigua que los intérpretes de uno de los litigios más importantes por el cacicazgo principal de Leymebamba y Cochabamba eran hablantes de castellano y quechua. En el documento del 25 de marzo de 1574 se lee que «el señor corregidor tomo e rrecibio juramento por Dios nuestro señor por la señal de la cruz en forma de derecho de todas dichas lenguas yenterpretes, que son ladinos en lenguas castellana yen la del Ynga...» (Transcripción de Schjellerup, 2005: 583). 
permitido conocer algunas características de este complejo idiomático del cual se resalta, por un lado, su tendencia de raíz monosilábica presente en la composición de antropónimos por la repetición de una misma sílaba como Pispis, Samsam, Solsol; y, por otro lado, su sistema toponímico caracterizado por las terminaciones -gat, -lap, -lon, -mal y -huala. Estas evidencias han sido vinculadas con una cultura local denominada chachapoya y algunos investigadores han sostenido la tesis de una «lengua chacha» (Cf. Torero 1989) o una lengua de los antiguos chachapuya (Cf. Taylor 1990) que representaría esta relación.

Sin embargo, mientras la arqueología y etnografía han podido comprobar la existencia de diversos rasgos comunes para todo el ámbito de la cultura chachapoya, la tesis de una lengua común y propia de la antigua cultura todavía carece de fundamento. Por lo tanto, en este artículo, se indaga el vínculo propuesto entre la cultura chachapoya y el sistema linguístico que dejó, principalmente, los vestigios toponímicos aún presentes en la zona. Para cumplir este fin, los objetivos que se persiguen son dos: por un lado, comprobar la pertenencia de las terminaciones toponímicas propuestas con la cultura chachapoya $y$, por otro lado, reunir evidencia en cuanto al significado de las terminaciones toponímicas registradas. El artículo está organizado en cuatro secciones: en $\S 1$ se presentan los antecedentes del trabajo de investigación, en §2 se detalla la metodología de investigación, en $\S 3$ se presentan los resultados del trabajo de campo y $\S 4$ se analizan los datos obtenidos.

\section{Antecedentes}

Los principales antecedentes de investigación linguística que proponen la existencia de una lengua distinta al castellano y el quechua en el sur de Amazonas están basados en datos obtenidos de la toponimia y la antroponimia local. En el campo de la toponimia, el acercamiento de Langlois (1939) ofrece la primera observación de las terminaciones toponímicas -ate, -on y -mal. De estas, las dos primeras son luego reanalizadas como -gat y -lon por otros investigadores, y el componente -mal permite al autor vincular una posible lengua extinta de esta región del Perú con alguna otra lengua del Yucatán a través de una comparación intuitiva, sin ningún tipo de análisis riguroso, entre los topónimos del valle del Utcubamba como Panamal, Ocumal, Osmal, Cuémal y los topónimos de la península del Yucatán como Uxmal, Itzamal, Chetumal, Ulumal, Cuzumal (Cf. Langlois 1939: 99). 
En esta misma línea, un trabajo más metódico propone un área idiomática chacha basada en la recurrencia de las terminaciones toponímicas $-\operatorname{lon}^{2}$, -mal y -lap. De acuerdo con las observaciones y análisis de Torero (1989), esta área idiomática se distribuiría por las provincias de Bagua, Bongará, Chachapoyas, Luya y Rodríguez de Mendoza en el sur de Amazonas con un lindero natural en el río Marañón, que lo limitaba con otras áreas como la DEN y la CAT propuestas también por el mismo autor como se muestra en la figura 1.

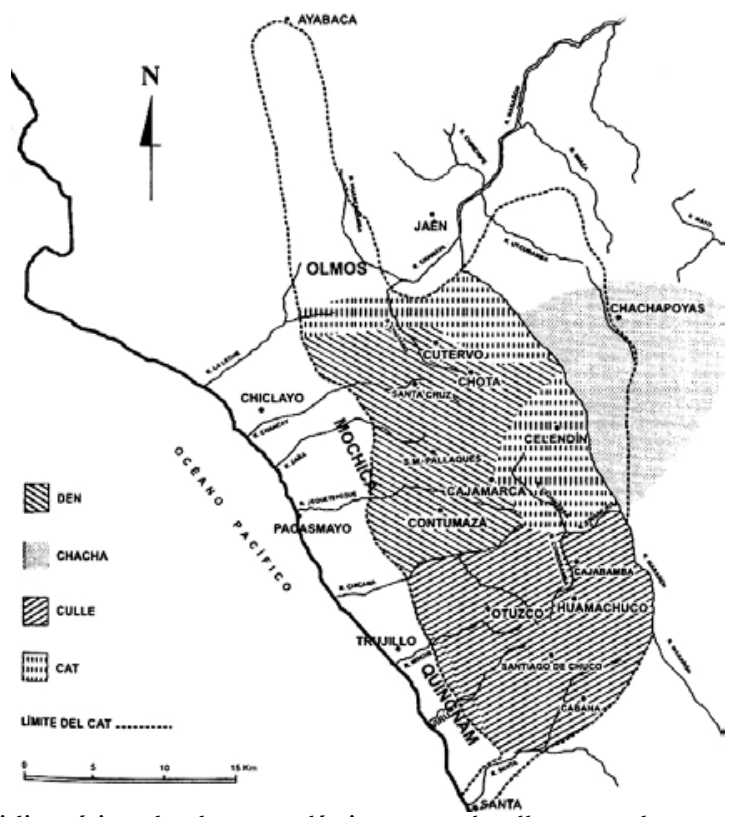

Figura 1. Área idiomática chacha y sus límites con el culle, cat y den.

Fuente: Torero (1989: 222)

Con trabajos de campo en la zona, Taylor (1990) condensa las propuestas anteriores en un sistema toponímico de lo que él denomina «lengua de los antiguos chachapuya» compuesto por las terminaciones -mal, -gat, -lap y -huala. A diferencia de los autores citados anteriormente, este investigador no incluye el componente -lon, pero sí añade otro con la forma -huala. Lo más resaltante de su trabajo es la asignación de los posibles significados de la terminación -mal como 'llanura o pampa'; -gat, 'río o agua'; -lap, 'fortaleza o pueblo fortificado' y -huala, 'cerro'.

2 Langlois había detectado la terminación -on que en muchos casos podría haberse segmentado como -lon, según el ejemplo mostrado en Chui-lon. 
Como hipótesis de trabajo se podría investigar la difusión de algunas terminaciones dentro y fuera de la zona considerada históricamente como chachapuya, por ejemplo, gat, gate, gache, cache, gote (ivariantes?), cuyo valor léxico podría ser 'río' o 'agua' (cf. shíngache (La Jalca), gache (Conila), jamingate (Olto), tóngate (Colcamar), gollongate (Santo Tomás), nombres de ríos); mal, de lejos la terminación más difundida entre los topónimos y cuyo sentido podría ser 'llanura', 'pampa' (existe un topónimo de Olleros, yulmal, cuya primera parte yul, es el nombre de un árbol local que aparece frecuentemente entre los nombres chachapuyas); lap, lape es la terminación de varios grupos de ruinas antiguas: conilape, yálape, cuélap y podría designar una fortaleza o un pueblo fortificado; huala significa tal vez 'cerro' (cf. shukahuala, nombre de un cerro, shuka significa 'gallinazo' en el quechua local; huala huala es el nombre de una cordillera). (Taylor 1990: 133).

En el campo de la antroponimia, el listado de 645 apellidos de «indios chachapoyanos» registrado por Zevallos Quiñones (1966) es sin duda una de las mejores evidencias sobre los apellidos locales con registro más antiguo en la zona. Los documentos utilizados datan, en su mayoría, de fines del siglo XVI y su origen corresponde a diferentes zonas donde estuvieron asentados los pueblos chachapoya o fueron llevados como mitmas. Una de las principales observaciones identificadas por este investigador es la peculiaridad de los antropónimos chachapoya que contrastan con otros de lenguas como el mochica, el culli, el quechua o el aimara.

Comparando este material con apellidos indígenas provenientes de otras partes del Perú, donde antiguamente se hablaron otras lenguas y dialectos, contrasta el fuerte exotismo de los nombres chachapoyas, que en su mayoría no presenta conexión alguna con los de las lenguas mochica, culli, cauqui y las de Piura; si descontamos un minúsculo porcentaje de quichuismo incaico y otro de origen yunga costeño originado por el régimen de mitimaes instalados, tampoco aparece relación con las lenguas quechua y aymara (Zevallos Quiñones, 1966: 4).

Otra observación se relaciona con los componentes iniciales de los apellidos que estaban constituidos por coll, cui, cul, ulla, chan, chec, chi, ga, gua, hoc, lla, oc, sub y ton, y los componentes finales en donde resaltan las formas al, el, $u l, d a, e t, i t, o t$. Según el análisis del autor, el uso del componente hoc- parece privativo del género femenino, ya que este se aplica regularmente en los nombres de mujeres.

En un análisis más linguiístico de estos antropónimos, Taylor (1990) sostiene que esta lengua era de raíz monosilábica, ya que varios de los apellidos están compuestos por una sola sílaba como Cam, Det, Dop, Oc, Pul, Sup, Yus, Yull, y los que contienen más de una parecen ser un conjunto de varios elementos 
monosilábicos como Acac, Huchuc, Pispis, Samsam, Solsol. Por otra parte, el autor presenta las posibles formas canónicas de la composición silábica basadas en los apellidos, a saber, VC, CV, CVC, CGV, CVG, CGVC, CVGC, en las que G representa las semivocales $w$ y $j$. De acuerdo con sus indagaciones, Taylor propone que la lengua poseía por lo menos cinco vocales /i/, /e/, /a/, /o/ y /u/, y que su sistema consonántico se clasificaba en veintiún posibles segmentos como se muestra en la tabla 1.

\begin{tabular}{|c|c|c|c|c|c|}
\hline Oclusivas sordas & $/ \mathrm{p} /$ & $/ \mathrm{t} /$ & & $/ \mathrm{k} /$ & \\
\hline Sonoras & $/ \mathrm{b} /$ & $/ \mathrm{d} /$ & & $/ \mathrm{g} /$ & \\
\hline Nasales & $/ \mathrm{m} /$ & $/ \mathrm{n} /$ & $/ \tilde{\mathrm{n}} /$ & & \\
\hline Laterales & & // & $/ \mathrm{I}^{\mathrm{y}} /(\mathrm{LL})$ & & \\
\hline Vibrante & & $/ \mathrm{r} /(?)$ & & & \\
\hline Fricativas sordas & $/ \mathrm{f} /$ & $/ \mathrm{s} /$ & $/ \mathrm{sh} /(\mathrm{X})$ & & $/ \mathrm{h} /(?)$ \\
\hline Sonoras & /b/ (V,B?) & & & & \\
\hline Africadas & & /c/ (TZ?) & $/ \mathrm{ch} /(\mathrm{CH})$ & & \\
\hline Semivocales & $/ \mathrm{w} /(\mathrm{V}, \mathrm{U} ?)$ & & $/ \mathrm{y} /$ & & \\
\hline
\end{tabular}

Tabla 1. Consonantes de la lengua de los chachapoya.

Fuente: Taylor (1990: 127)

\section{Metodología e instrumentos}

\subsection{Determinación del objeto de estudio}

Si bien algunos antecedentes revisados han postulado que los vestigios toponímicos y antroponímicos presentados en $\$ 1$ pertenecerían a una lengua extinta denominada chacha o se ha propuesto su vínculo con los antiguos chachapoyas, resulta necesario confirmar esta supuesta conexión entre los datos linguíísticos y la cultura local que se desarrolló en esta parte del departamento de Amazonas. Para lograr este fin, utilizamos los antropónimos recopilados por Zevallos Quiñones (1966), ya que este documento consigna el sistema tradicional de transmisión de apellidos que se seguía aplicando a inicios de la época colonial por los diferentes pueblos que pertenecían a la cultura chachapoya.

Un primer cotejo entre los vestigios toponímicos y los antropónimos chachapoya indica que, de las terminaciones toponímicas -gat, -mal, -lon, -huala y -lap, propuestas como de la lengua que hablaron los chachapoyas (Taylor, 1990), solo -gat (con sus variantes entre ellos -cat) y -mal están presentes en

3 Un total de 11 antropónimos registrados termina con alguna variación de -gat y un total de 9 en $-m a l$. 
ambos casos. De la comparación hecha, se registra un solo apellido con terminación -guala (quizás -huala), no se constata la presencia de la terminación -lon, de cierta frecuencia en la toponimia de la zona chachapoya, y solo se atestiguan dos variaciones en cuanto a la terminación -lap, que aparece en las formas de -lap y -llap ${ }^{4}$.

Por datar de los siglos xVI y xVII, los antropónimos están escritos con una grafía cercana a la pronunciación nativa del lugar, lo que resulta interesante sobre todo para el caso de -gat, que presenta variantes similares a las toponímicas, como -gache, -gate y -cate, pero también registra varias formas sin vocal -e al final como -cat, -cot y -gat. Estas últimas formas no presentan correspondencia con las terminaciones toponímicas. ${ }^{5}$

(1) Listado de antropónimos que terminan en -gat registrado por Zevallos Quiñones (1966)

$\begin{array}{ll}\begin{array}{l}\text { Changot } \\ \text { Gochegat }\end{array} & \text { (Yuya, 1599; Yapa, 1587) } \\ \text { Gullacot } & \text { (Yapa, 1587) } \\ \text { Gamgache } & \text { (Yapa, 1587) } \\ \text { Muchagache } & \text { (Yapa, 1587) } \\ \text { Muagate } & \text { (Yapa, 1587) } \\ \text { Hochcat } & \text { (Leymebamba y Balsas, 1735) } \\ \text { Hochgate } & \text { (Leymebamba y Balsas, 1735) } \\ \text { Pochcate } & \text { (Yapa, 1587) } \\ \text { Huchcat } & \text { (Leymebamba y Balsas, 1735) } \\ \text { Yancot } & \text { (Yapa, 1587) }\end{array}$

En el caso de la terminación -mal, al igual que en los topónimos, no se constata variación alguna en los antropónimos.

(2) Listado de antropónimos terminados en -mal registrado por Zevallos Quiñones (1966)

Puymal (Chachapoyas, 1598)

Sichmal (Gopara, 1597)

4 Solo dos antropónimos terminan en -lap, mientras existen cinco apellidos con la terminación -llap, tres de ellos presentan incluso la terminación -mallap.

5 En la investigación toponímica se ha registrado tan solo un caso de terminación en consonante: Cuchcat, en el distrito de La Jalca (Chachapoyas). 


\author{
Xazmal (Gopara, 1597) \\ Yasmal (Gopara, 1597) \\ Olmal (Leymebamba y Balsas, 1735) \\ Paymal (Leymebamba y Balsas, 1735) \\ Sanacmal (Gopara, 1597) \\ Citimal (Gopara, 1597)
}

Estas observaciones no solo constituyen un indicio para asegurar por ahora la pertenencia de las formas linguísticas -gat y -mal al idioma usado por los chachapoyas, sino que, por su alta frecuencia entre los antropónimos, también confirman la selección de estas dos terminaciones toponímicas para su estudio en la zona de influencia de esta cultura. En ese sentido, -gat y -mal se convierten en nuestras dos terminaciones toponímicas vinculadas con la cultura chachapoya.

\title{
2.2 Determinación del área de estudio
}

Después de comprobar que estas dos terminaciones toponímicas están relacionadas con la cultura chachapoya, como se ha visto anteriormente en la comparación antroponímica, queda pendiente establecer su relación en el ámbito territorial con los pueblos chachapoya. En esta línea, las investigaciones histórico-arqueológicas realizadas hasta la fecha en los Andes y Amazonía del nororiente del Perú, aunque todavía lejos de ser exhaustivas, han logrado definir el área antiguamente habitada por la cultura preincaica de los chachapoyas. Aunque eran pueblos independientes, las diferentes características compartidas entre todos los grupos, entre ellas patrones arquitectónicos ${ }^{6}$, rasgos culturales ${ }^{7}$ y técnicas agrícolas ${ }^{8}$, han permitido clasificar a las poblaciones que habitaban la zona en cuestión como pertenecientes a una misma cultura (Schjellerup, 2005; Kauffmann y Ligabue, 2003).

6 P. ej. las emblemáticas casas redondas (Koschmieder 2012: 13).

7 Como el culto de cabezas trofeo, el cual se practicaba desde la zona norte, p. ej., Putquerurco (Lámud), hasta la zona sur, p.ej. La Petaca (Leimebamba), y la producción de textiles de alta calidad (Koschmieder 2012: 15).

8 Lerche (1986: 40-41). 


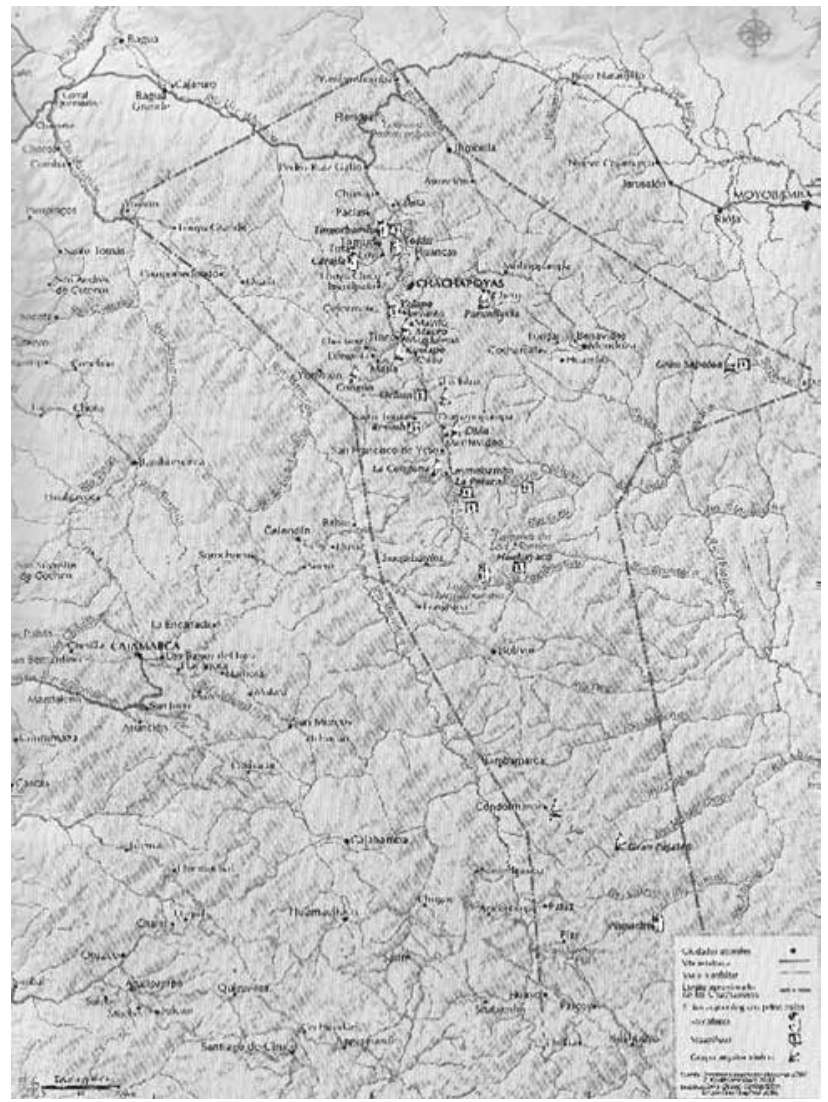

Figura 2. Extensión comprobada de la cultura chachapoya.

Fuente: Kauffmann y Ligabue, 2003: 23.

En su apogeo, dicha cultura llegó a ocupar gran parte del territorio del actual departamento de Amazonas, además de sectores colindantes de los actuales departamentos de La Libertad y San Martín (véase la figura 2). Una primera revisión de los registros disponibles ${ }^{9}$ con respecto a las formas toponímicas -gat y -mal en este espacio territorial, demuestra que las dos terminaciones presentan una alta frecuencia dentro del territorio con evidencias arqueológicas de la cultura chachapoya ${ }^{10}$. La terminación toponímica -mal no presenta

9 INEI, DIRCETUR Amazonas, diversas investigaciones arqueológicas (véase la bibliografía).

10 Tomando por referencia los datos del SIGMED (centros poblados), ING (topónimos diversos) y MINEDU (cerros y ríos) por departamentos, frente a 69 topónimos con terminación en -mal en 
variación en su forma, aparece con muy poca frecuencia fuera de este territorio $y$, hasta el momento, no ha sido relacionada con otras lenguas. Caso distinto se da con la terminación toponímica -gat, que al parecer presenta variación en su forma, pues aparece con frecuencia relativa en otros espacios no chachapoyas, y se vincula con otras posibles lenguas colindantes.

Como se ha observado en la figura 1, Torero (1989), aparte del área idiomática chacha, propone un área idiomática CAT que abarca distintas provincias de los actuales departamentos de Cajamarca, Amazonas, La Libertad y Lambayeque. Plantea el área CAT como un territorio toponímico marcado por el segmento final -cat o -cot y sus variantes -gat y -got apoyados por una $e$ final en varias provincias de los departamentos mencionados y con alta frecuencia en Cutervo, Cajamarca, Celendín, Contumazá, Luya, serranías de Ferreñafe y vertientes marítimas de Chota. Una de las variantes en el área CAT, -gat, es semejante a la forma atestiguada en el área chacha (Cf. Taylor, 1989) incluso con un significado similar. Esto, por un lado, descarta la posibilidad de identificar la terminación -gat y variantes como una característica exclusiva del idioma chacha, y por lo tanto una herramienta adecuada para delimitar con seguridad el área idiomática del chacha. Por otro lado, evidencia la necesidad de comprobar su pertenencia, sea de préstamo u originaria, a la lengua en cuestión, así como de identificar sus variantes en la zona.

Por lo expuesto arriba, por una parte, queda bastante clara la relación de la terminación toponímica -mal con el ámbito territorial chachapoya y, por otro lado, se levanta una controversia con respecto a la terminación -gat y sus variantes. Si bien se puede confirmar su relación con la cultura chachapoya, tanto por su vínculo con los antropónimos chachapoya como también por su presencia en el territorio chacha, emerge una nueva pregunta sobre la relación de esta terminación toponímica con la cultura chachapoya y otras del área toponímica CAT que propone Torero.

\subsection{El topónimo como signo lingüístico}

La controversia en torno a -gat y variantes como -cat (en referencia al área CAT y chacha) demanda comprobar, en primer lugar, su pertenencia al ámbito territorial chachapoya y, en segundo lugar, a identificar sus variantes posibles en dicha zona. Con este objetivo, la toponimia, como ciencia que estudia los nombres de los lugares geográficos entendidos aquí como topónimos y, por

Amazonas, se encuentran tan solo 9 en Cajamarca, al igual que en Piura, 3 en La Libertad, 2 en San Martín y 1 en Lambayeque. 
extensión, los sistemas de denominación elaborados por los grupos humanos (Cf. Solís, 1997), se convierte en el marco teórico que guiará el presente estudio. Este marco en particular resalta entre sus disciplinas fundamentales tanto a la linguística como a la filología (Cf. Cerrón-Palomino, 2015) y el estatus del primero se le asigna en la medida que esta última formaliza el estudio de los topónimos como signos linguí́sticos.

Entendido un topónimo como la asociación entre un significante y un significado, este responde a una categoría gramatical denominada nombre y puede constituirse como una palabra, una frase u oración nominalizada. En el significado, los topónimos están sugeridos socialmente por una serie de motivos, por ejemplo, los geográficos como sucede en los topónimos con la terminación -gat en su variante -gach, p.e., Singache ['sin.ga.fe] y Tongache ['ton.gatfe] en la Jalca Grande (Chachapoyas) que hacen referencia a un río y un pozo, respectivamente. Al parecer, el componente común de estos topónimos -gache se asocia con el significado de 'recurso hídrico'. En el significante, el topónimo está compuesto por unidades fonológicas que pertenecen a una lengua específica. En una primera aproximación, el componente -gache 'recurso hídrico' parece estar compuesto por cuatro unidades fonológicas que forman parte de la morfología de una lengua, a saber, /-gatee/. Esta terminación toponímica /-gatfe/ con el posible significado 'recurso hídrico', al añadirse a los otros componentes aquí presentados como ejemplos, sin-gatfe y ton-gate, conforma una categoría gramatical denominada nombre. Por hacer referencia a lugares geográficos, a estos nombres se los denomina topónimos.

Hay que tomar en cuenta que los topónimos no solo están sugeridos por el contexto geográfico o ambiental como se ha ejemplificado arriba, sino que estos también pueden estar motivados por hechos históricos o culturales (Cf. Cerrón-Palomino, 2015; Chávez, 2003; Solís, 1997). Para Solís (1997), existen dos tipos de topónimos: los reales, que se relacionan con la naturaleza donde se desenvuelve el ser humano, como las configuraciones geográficas o relieve, las propiedades físicas del terreno, el cromatismo, los recursos zoológicos, botánicos, hidrológicos, etc., y los ideales, que resultan de la creatividad cultural y se relacionan con asuntos honoríficos, divinidades, personajes históricos, sucesos históricos. En este sentido, en la medida en que interviene el afán nomenclador del hombre cuando entra en relación con el espacio que desea ubicar socialmente, la relación entre el significante y el significado de un topónimo no es arbitraria y responde a motivaciones socioculturales en especial.

Los nombres de lugares no son pura arbitrariedad, más o menos caprichosamente impuestos por el hombre, muchos de estos nombres son 
intentos de explicación de la naturaleza espontánea que presentan los espacios geográficos, sugieren su propia denominación ya porque el hombre cree que los lugares desarrollan acciones tales como podemos observar en wiñaq o qellayqucha; en otros, el hombre entiende cómo los lugares tienen o poseen cosas tal cual se observa en puyuyuq, uqshapampa. (Chávez 2003: 9-10).

\section{Resultados}

\subsection{Terminación toponímica -gat(e)}

La terminación toponímica aquí representada como -gat(e) posee, hasta donde se ha podido registrar, las variaciones -got(e), -cat(e), - $\cot (e)$ y $-\operatorname{gach}(e)$. Como se ha revisado anteriormente, tanto Langlois (1939) como Taylor (1990) ya habían observado este componente toponímico en el territorio de estudio y fue este último quien propuso como hipótesis de trabajo el significado de 'río' o de 'agua' para este componente final. Taylor sustentó su postura sobre la base de topónimos que designaban a ríos como Shíngache, Gache, Jamingate, Tóngate y Gollongate.

Sobre su significado, los pobladores actuales no reconocen algún sentido de los nombres de estos lugares; sin embargo, el registro de una muestra de estos topónimos en los distritos de la Jalca Grande (provincia de Chachapoyas), Lámud, San Cristóbal de Olto y Colcamar (provincia de Luya) ha confirmado una motivación geográfica principal por una 'quebrada', un 'pozo' o un 'río'. Por lo tanto, en (3), -gate, -cate, -gote y -gache sugieren el significado de 'recurso hídrico' en esta terminación toponímica.

$\begin{array}{lll}\text { (3) Yaquin-gate } & \text { 'pozo' } & \text { Lámud, Luya } \\ \text { Suitin-gate } & \text { 'río' } & \text { Lámud, Luya } \\ \text { Huasin-gate } & \text { 'río' } & \text { Lámud, Luya } \\ \text { Jamin-gate } & \text { 'quebrada' } & \text { Olto, Luya } \\ \text { Ol-cate } & \text { 'pozo' } & \text { Colcamar, Luya } \\ \text { Yal-cate } & \text { 'quebrada' } & \text { Colcamar, Luya } \\ \text { Pen-gote } & \text { 'pozo' } & \text { Jalca Grande, Chachapoyas } \\ \text { Sín-gache } & \text { 'río' } & \text { Jalca Grande, Chachapoyas } \\ \text { Tón-gache } & \text { 'pozo' } & \text { Jalca Grande, Chachapoyas }\end{array}$


Yaquingate.- [ya.kin.' ga.te], pozo, Cuémal, Lámud.

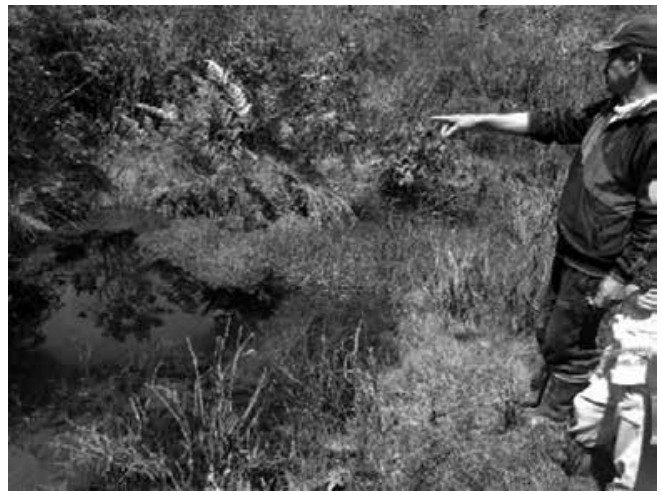

Olcate.- [ol.'ka.te], quebrada, Colcamar

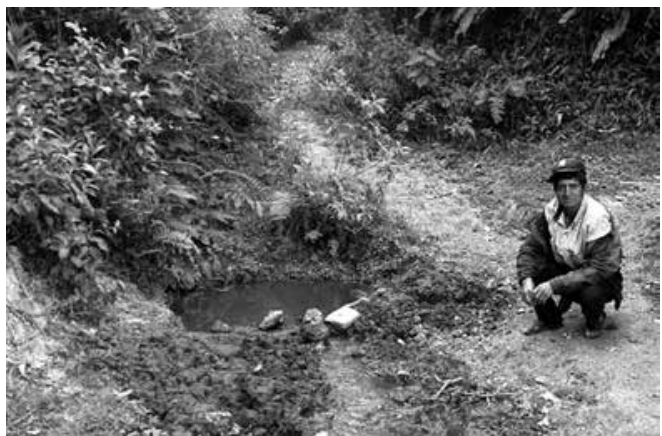

Figura 4. Ejemplos de sitios con terminación toponímica en -gat, identificados en el trabajo de campo. Fotografías: J. Valqui.

En el significante, esta terminación toponímica que varía en -gate, -cate, -gote y -gache, parece provenir de una construcción fonológica anterior que no poseía la vocal [e]:/-gat5/, como también se ha confirmado en la antroponimia ya analizada anteriormente, donde no aparece regularmente tal vocal. La aparición del segmento -e se puede explicar como producto de una inserción por un sistema linguíístico ajeno a la lengua originaria, el del castellano. Si este análisis fuera correcto, se puede suponer un estadio anterior de este componente toponímico carente de la vocal mencionada $\left(\right.$-gate $<$-gat). ${ }^{11}$

11 Solo existe un caso, Cuchcat en el distrito de La Jalca (Chachapoyas) que no presenta la característica -e final de la terminación en cuestión. 
Por otra parte, se observa que cuando el componente toponímico en cuestión es precedido por una consonante nasal, esta terminación siempre presenta una consonante velar sonora $[g]$, lo que no ocurre en el caso de que le preceda una consonante diferente. La alternancia exclusiva después de las nasales permite la deducción de un estadio más antiguo en común de estas variaciones, iniciado por una consonante velar oclusiva sorda (gat $<k a t)$. Tomando en cuenta los antropónimos más próximos a la pronunciación original, y en vista de las evidencias sobre el componente -gat en territorios vecinos al idioma chacha, se puede reconstruir a \%/kat/ como la forma más originaria.



Figura 5. Distribución toponímica de -gat(e),-got(e), -cat(e), -gach(e) en el departamento de Amazonas.

Fuente: elaboración propia. 
Con esta base en común, las cuatro alternancias se explicarían linguiísticamente por una serie de procesos fonológicos que sonorizaron la forma reconstruida como \%/-kat/ y la palatalizaron, en algunos casos, cuando se insertaba la vocal -e. Sin embargo, existen topónimos que presentan esta última forma sonorizada y palatalizada gach sin que le preceda alguna consonante, como el caso del río Gache en Lámud, pero el escaso material no permite llegar a más conclusiones fonológicas sobre este punto.

Por lo pronto, se puede concluir que las terminaciones toponímicas -gat(e),got(e), -cat(e), -gach(e) significan 'recurso hídrico'. Su recurrencia por distintos pueblos de las provincias actuales de Chachapoyas y Luya nos revela que este componente final fue utilizado como parte de un sistema linguiístico común a estos territorios.

También existen casos raros de topónimos con este tipo de terminación que no se han podido relacionar con algún recurso hídrico. En estos casos, se debe considerar la posibilidad de que la pérdida de inteligibilidad del topónimo por la desaparición del idioma de origen haya ocasionado modificaciones en el uso de los topónimos frente a los sitios referidos, como sucede por ejemplo en el caso de Léngache (distrito de Lámud, provincia de Luya). Actualmente, el topónimo designa a un sitio funerario de la cultura chachapoya conocido por los pobladores de la zona, pero al mismo tiempo se refiere a una laguna ubicada en la cercanía del sitio arqueológico, por lo que se puede asumir que el topónimo Léngache pasó de solo designar a la laguna a usarse también de referencia para los restos arqueológicos, los cuales se hicieron conocidos finalmente bajo este mismo nombre.

\subsection{Terminación toponímica -mal}

La terminación -mal presenta más frecuencia que la terminación toponímica anterior. Hasta donde se ha visto, no ha sufrido alternancia alguna desde su primera documentación en los documentos coloniales más antiguos, y tampoco presenta variaciones ni en la toponimia ni la antroponimia actuales de la zona.

Taylor (1989) propuso el significado de 'llanura o pampa' para el componente -mal. Él encontró que el topónimo Yulmal (Olleros, Chachapoyas) provenía de Yul, el nombre de una planta local, y dedujo el significado del segundo componente como 'llanura o pampa'. Los pobladores actuales de las provincias estudiadas no identifican el significado de las denominaciones toponímicas compuestas por -mal. Muchos interpretan desde el castellano una carga negativa del topónimo y lugar por su componente -mal. Así, por ejemplo, el topó- 
nimo Gulmal es, según muchos, un 'lugar donde había un gulgul (pavo) malo' y Cochamal (provincia de Rodríguez de Mendoza) sería una 'laguna (cocha en quechua) mala'.

El registro de una muestra de estos topónimos en los distritos de la Jalca Grande y Quinjalca (provincia de Chachapoyas), Lámud, Trita y Colcamar (provincia de Luya), como se muestra en (4), nos provee una amplia gama de ejemplos de la terminación toponímica aludida, como referencia para la comprobación o refutación de las propuestas de significado de -mal.

\begin{tabular}{lll} 
(4) Go-mal & 'pampa' & \multicolumn{1}{c}{ Jalca Grande, Chachapoyas } \\
Os-mal & 'cerro, pampa' Jalca Grande, Chachapoyas \\
Yu-mal & 'ladera' & Jalca Grande, Chachapoyas \\
Shil-mal & 'pampa' & Quinjalca, Chachapoyas \\
Cho-mal & 'pampa' & Quinjalca, Chachapoyas \\
Duer-mal & 'pampa' & Quinjalca, Chachapoyas \\
Chil-mal & 'laguna' & Cuemal, Luya \\
Gul-mal & 'montaña' & Cuemal, Luya \\
Sol-mal & 'ladera' & Trita, Luya \\
Shul-mal & 'ladera' & Trita, Luya \\
Gash-mal & 'montaña' & Colcamar, Luya \\
Puy-mal & 'cerro' & Colcamar, Luya
\end{tabular}

En su conjunto, en el trabajo de campo, no se ha determinado con absoluta seguridad el significado de -mal, ya que, como se observa en (4), muchos topónimos examinados designan a pampas, laderas o montañas, de manera que no se puede descartar ninguno de los significados propuestos en investigaciones anteriores. Además, en casi todos los casos, el primer componente del topónimo es imposible de interpretar, a excepción de algunos topónimos aislados: los raros casos de composición de un topónimo por un primer componente quechua y una terminación chacha ${ }^{12}$, y los casos de componentes aparentemente derivados de los nombres de plantas conservados hasta la actualidad. En resumen, sin embargo, no todos los sitios visitados en el marco del trabajo de campo están relacionados con una pampa o llanura, por lo que se debería considerar una interpretación alternativa de esta terminación toponímica. Los casos investigados con un primer componente inteligible hacen suponer el significado de 'lugar donde abunda un determinado recurso' para -mal.

12 Ver también capítulo 5.3. 
Gachmal.- [gaf.' mal gatf.' mal], montaña donde nacen quebradas, Colcamar

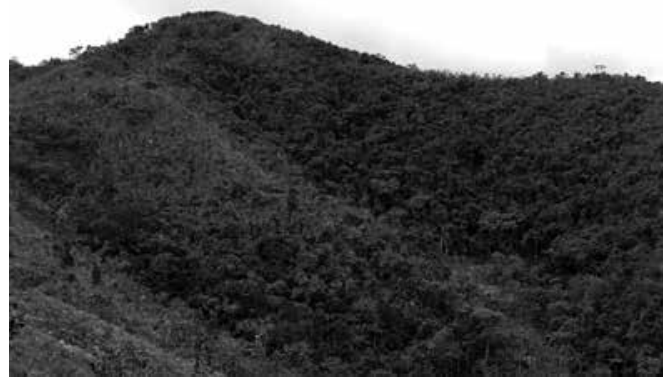

Shilmal.- [ ill.'mal], ladera, Quinjalca

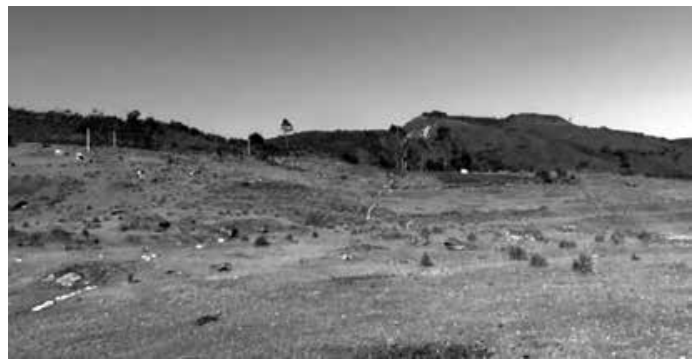

Figura 6. Ejemplos de sitios con terminación toponímica en -mal, identificados en el trabajo de campo.

Fotografías: J. Valqui.

El indicio más notorio de esta interpretación es el topónimo Gachmal (Colcamar, Luya), que puede estar compuesto por gaf $\sim$ gat $<$ gat(e) $<$ kat 'recurso hídrico' y -mal 'lugar donde abunda un determinado recurso'. De hecho, Gachmal es una montaña ubicada en las alturas de Colcamar, en cuyos dos lados nacen quebradas, una que irriga a Inguilpata y otra a Colcamar. Así mismo, el caso de Mashumal (Huancas, Chachapoyas), topónimo compuesto por un primer morfema quechua -y por lo tanto entendible- y una terminación chacha, inclina la interpretación más a favor de 'lugar donde abunda un determinado recurso': Mashumal Mashmal, conformado por la palabra quechua mashu 'murciélago' (Cf. Taylor, 2006) y la terminación -mal, designa a un complejo de cavidades de hasta cuatro metros de profundidad cuyo rasgo preponderante es la abundancia de murciélagos, por lo que es conocido entre los pobladores de Huancas; las cavidades incluso presentan restos de incendios que los habitantes del lugar habrán causado para ahuyentar a los murciélagos. Por designar 
a cavidades, en este caso es difícil establecer una relación con alguna pampa o llanura.

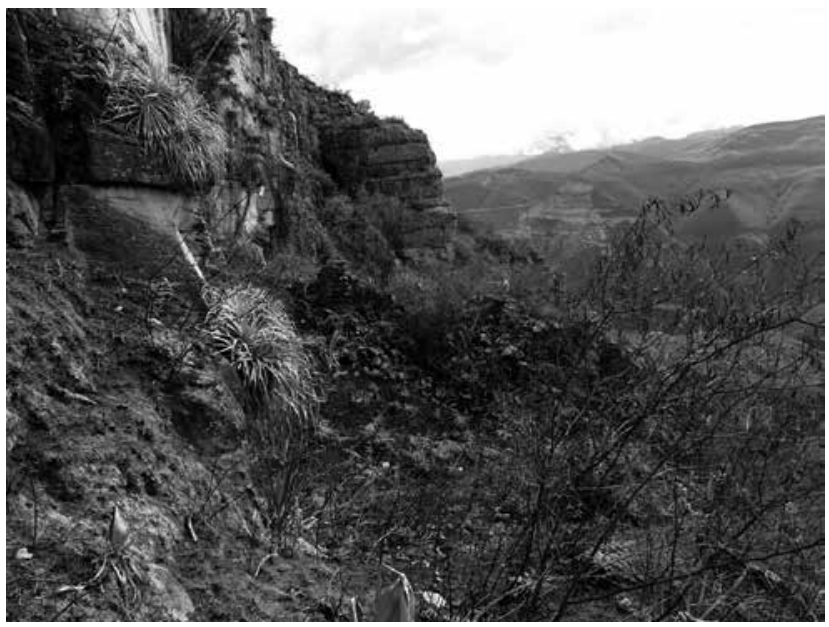

Figura 7. Mashumal (Huancas, Chachapoyas). Fotografía: M. Ziemendorff

Además, algunos topónimos como Gulmal en Cuémal, Lámud (provincia de Luya) o Shilmal en Quinjalca (provincia de Chachapoyas) parecen provenir en su primer componente de plantas locales como gulgul y shilshil, cuyos nombres ancestrales se han conservado hasta hoy. Algunos habitantes de las zonas mencionadas identifican como primera característica de los lugares en cuestión la abundancia de la planta respectiva. Si esta interpretación fuera cierta, quedaría pendiente explicar por qué estos topónimos no recogen la duplicación del morfema respectivo que se manifiesta en los nombres de plantas gulgul y shilshil. ${ }^{13}$

13 Este fenómeno también se observa en el análisis de la relación entre antropónimos y topónimos: mientras Zevallos Quiñones (1966) registra el apellido Solsol, el topónimo Solmal (distritos de Levanto, Chachapoyas, y Olto, Colcamar y Trita, Luya) no presenta la duplicación de la sílaba sol. El apellido Subsolsol, en cambio, evidencia que la repetición de esta sílaba no impedía que se le agregue otra sílaba. 




Figura 8. Distribución toponímica de -mal en el departamento de Amazonas. Fuente: elaboración propia.

\subsection{Presencia de la forma Gach- en la toponimia local}

El registro toponímico ha revelado una cantidad considerable de topónimos que contienen la forma gach-, la cual es muy similar a la terminación toponímica -gach como variación de -gat. Más aún, el topónimo Gachmal (gach + mal) y el sitio designado por este, con presencia de varias fuentes de agua, constituye un indicio interesante que permite suponer que también los topónimos que inician por el componente gach- se relacionan con el significado de 'agua' y otros parecidos.

Esta forma presenta variaciones similares a la terminación toponímica -gat, además de tener menos variedad en cuanto a sus terminaciones. Las variacio- 
nes que se han podido identificar incluyen Gash-, Cash-, Cach-, Cas-, Cat-, Gat-, Gac-, así como Cosh- y Cot-. Aparte del topónimo Gachmal antes descrito, en el trabajo de campo se han podido identificar otros topónimos que inician por gach-, todos relacionados con el significado 'recurso hídrico', como Gache (Lámud), Gachac (San Cristóbal de Olto) y Cachamal (Quinjalca).

Gachac.- [ga.' tfax], cascada, San Cristóbal de Olto.

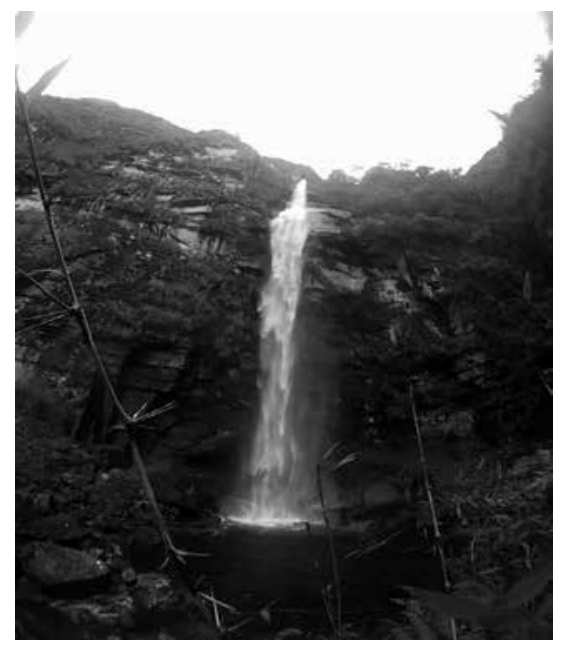

Gache.- ['ga.te], río, Lámud.



Figura 9. Ejemplos de sitios con topónimos que inician por gach-, identificados en el trabajo de campo.

Fotografías: J. Valqui, C. Laronde.

La distribución de los topónimos identificados, aunque más concentrada en el núcleo de la zona chachapoya, es similar a la recurrencia de los topónimos 
con terminación en -mal y -gat discutidos anteriormente, además de que es menos frecuente.

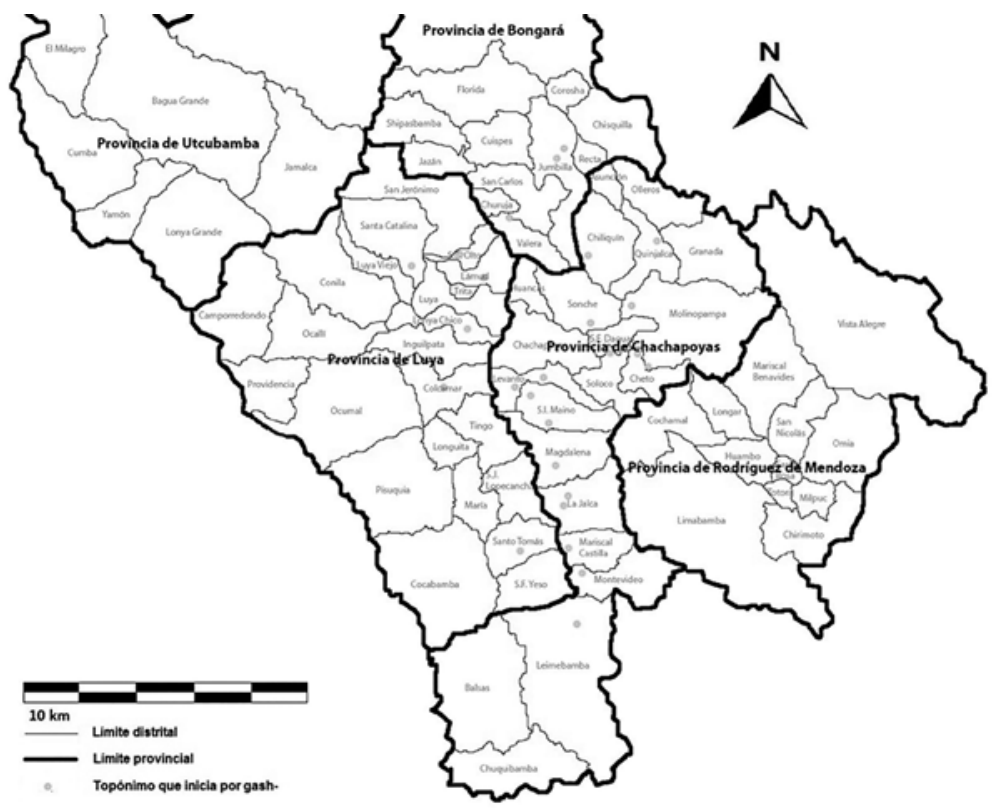

Figura 10. Distribución de topónimos que inician por Gach- en el departamento de Amazonas.

Fuente: elaboración propia.

\section{Análisis}

\section{1 Área idiomática según los morfemas toponímicos estudiados}

En la región Amazonas, la concentración máxima de los topónimos que contienen las formas estudiadas se observa en la provincia de Chachapoyas, con excepción de sus distritos sureños, y en el oriente de la provincia de Luya. En cambio, su frecuencia en las demás provincias con evidencia arqueológica, aunque escasa, de la cultura chachapoya, como Bagua, Bongará, Rodríguez de Mendoza y Utcubamba, es mínima. En el caso de -mal, los departamentos colindantes de Amazonas presentan una cantidad mínima de topónimos con esta terminación, mientras que, para el caso de gat-, como ya se ha mencionado 
anteriormente, se constata una alta frecuencia en los departamentos de Cajamarca y La Libertad. ${ }^{14}$

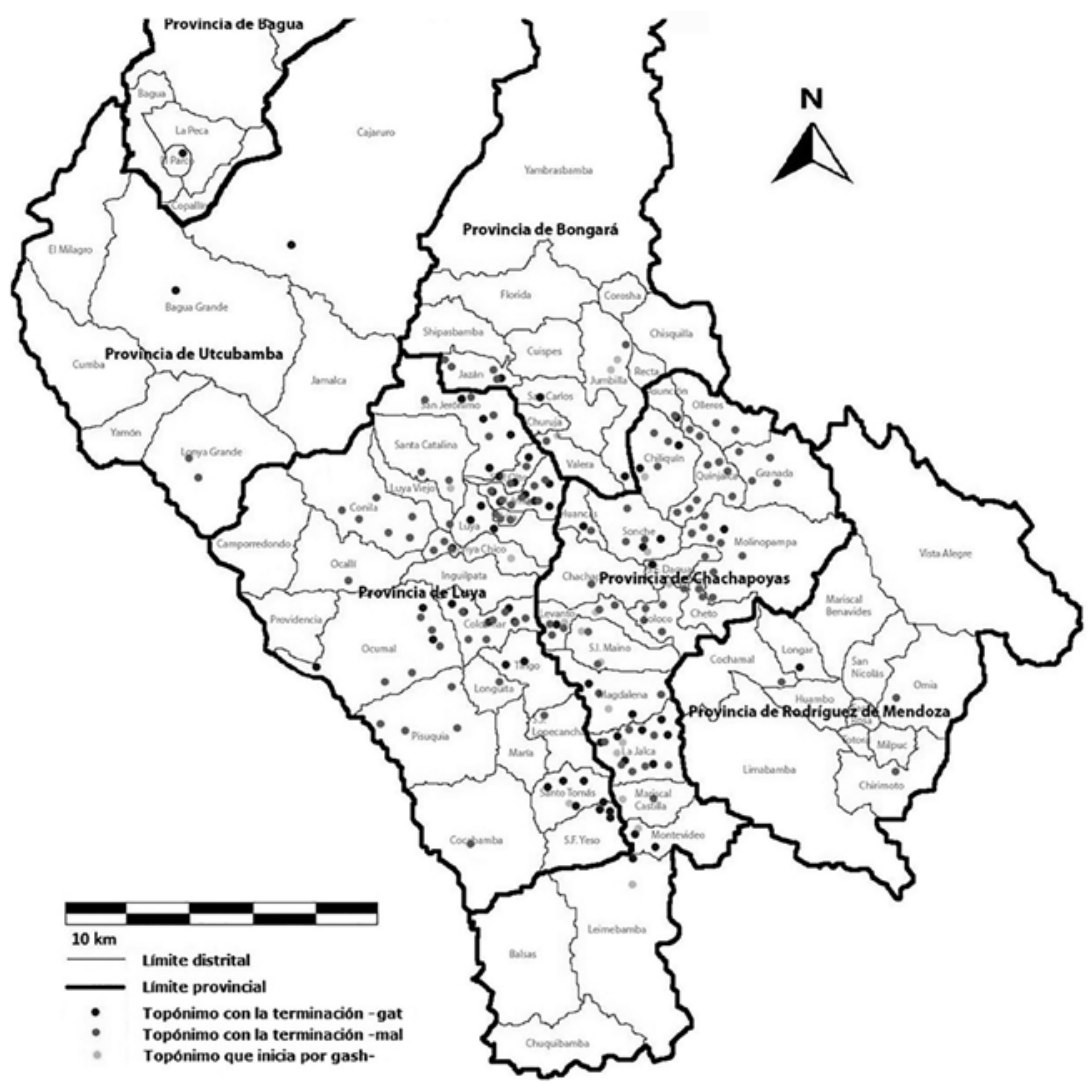

Figura 11. Distribución de topónimos con los morfemas -mal, -gat y gach- en el departamento de Amazonas.

Fuente: elaboración propia.

14 Si bien para la identificación de los topónimos que contengan los morfemas estudiados se han revisado fuentes diversas, para fines de comparación y evaluación de concentración de topónimos relevantes, se han tomado en consideración solo tres fuentes: SIGMED para centros poblados, IGN para topónimos diversos (excluyendo los centros poblados) y MINEDU (para cerros y ríos). 
Comparando el área delimitada por el uso y la frecuencia de los morfemas aludidos con la zona identificada como habitada por los pueblos chachapoyas por las investigaciones histórico-arqueológicas, se constata cierta correspondencia entre la evidencia linguística aquí estudiada y la cultura chachapoya. Sin embargo, el área toponímica representa solo la parte nuclear del territorio chachapoya propuesto por las investigaciones arqueológicas (cf. la figura. 2). Esta discrepancia se puede deber a que el registro toponímico se ha basado en fuentes actuales y que, por lo tanto, es muy probable que la zona determinada sobre la base de estos datos no corresponda a la distribución original del idioma al que pertenecen las terminaciones en cuestión. ${ }^{15}$

Por ahora, la consulta de fuentes históricas, como por ejemplo mapas y registros de los primeros siglos después de la conquista española de la zona, no ha aportado con mayores vestigios. Según dejan entender las fuentes del siglo XVI y XVII, al momento de la conquista, la lengua o las lenguas aquí atestiguadas ya no eran de uso predominante en la zona, de forma que el sistema toponímico del quechua -aparentemente el único idioma encontrado por los españoles en el ámbito chachapoya- ya había influido notablemente sobre la toponimia local, como ocurre ahora con el sistema castellano y los cambios de topónimos.

Ahora bien, por el momento, considerando la evidencia mostrada, se puede confirmar la existencia de una lengua común en los diversos pueblos chachapoyas que habitaban las actuales provincias de Luya y Chachapoyas, ya que no solo compartían las terminaciones toponímicas, sino también su significado que se ha podido comprobar para ambas provincias. Por tratarse de pueblos con diversos rasgos comunes, a pesar de algunas costumbres variadas ${ }^{16}$, es de suponer que los pueblos pertenecientes a la cultura chachapoya que radicaban fuera de esta área también pertenecían a la misma área idiomática, pero a nivel linguiístico. Esta hipótesis todavía se está por comprobar.

En nuestro estudio, como hemos mencionado anteriormente, las variantes con el sentido de 'agua' para la zona CAT propuesta por Torero, también aparecen en la zona de la cultura chachapoya, salvo la adición de la variante -gach

15 Además, en el presente estudio se han analizado tan solo dos morfemas toponímicos. Aunque se trate de los morfemas más notorios que se han podido identificar para el idioma chacha hasta la fecha, no pueden conformar una base suficientemente estable para formular conclusiones definitivas sobre la extensión del idioma en cuestión, ya que existen demasiados factores posibles que hayan podido influenciar el uso de dichas terminaciones toponímicas, aparte de la extensión misma del idioma chacha.

16 Por ejemplo, en cuanto a sus ritos funerarios, donde según el ámbito se usaban sarcófagos, mausoleos de diferentes materiales, nichos y cuevas. 
y la posibilidad de que un morfema raíz gach- aparezca en las denominaciones toponímicas en este espacio territorial. Por ahora, de los datos aquí estudiados y en relación con las hipótesis presentadas antes, se puede continuar con dos propuestas: 1 . Hay vestigios de que una lengua originaria común en este ámbito territorial, cuyo sistema toponímico indicaba con -gat la presencia del recurso hídrico y -mal la presencia o abundancia de algo como un recurso natural, fue desplazada ulteriormente por el quechua de acuerdo con los estudios históricos y la evidencia linguíística. Según este escenario, el topónimo Gachmal sería el ejemplo de un mismo sistema linguístico usado en el espacio territorial delimitado para la cultura chachapoya. 2. Estamos ante un escenario de préstamo linguístico, donde cronológicamente, solo se podría sugerir que la difusión de la forma */-kat/ fue anterior a la desaparición del idioma de los chachapoyas. Esta forma nos muestra un vínculo con pueblos ubicados más al oeste del territorio chachapoya.

\subsection{Otros aspectos de la lengua chacha}

Aparte de las conclusiones toponímicas de la lengua o lenguas en el territorio chachapoya, los resultados del presente trabajo permiten fortalecer algunas características del sistema linguiístico estudiado. En primer lugar, los topónimos revisados tanto en el trabajo de campo como también en la revisión de las diversas fuentes con registro de topónimos han proporcionado más pruebas para la hipótesis del carácter monosilábico del idioma de los antiguos chachapoya propuesto por Taylor (1990): una cantidad considerable de dichos topónimos se puede desagregar en componentes monosilábicos.

Especialmente los ejemplos de topónimos cuyo significado se ha podido identificar y que están compuestos por dos morfemas confirman esta característica del idioma: Gachmal, compuesto por los dos topónimos investigados en el presente trabajo gach y mal, así como Yul-mal y Shil-mal. Además de comprobar el carácter monosilábico del idioma chacha, los topónimos mencionados indican que el primer morfema de los topónimos parece tener un significado propio y concreto, el cual está en relación con el sitio denominado, de forma que existe la posibilidad, en investigaciones futuras, de identificar más palabras chacha a base del análisis cuidadoso de los topónimos identificados, habiendo ya definido el significado de la terminación respectiva.

Una segunda conclusión del trabajo realizado consiste en los indicios importantes de una época de coexistencia de los idiomas quechua y chacha en los territorios chachapoya. Aunque no hay evidencia que revele cuándo se dio 
este tiempo de existencia paralela, dejó topónimos compuestos por morfemas de ambas lenguas, como por ejemplo Mashu-mal (Huancas, Chachapoyas), Cocha-mal (Cochamal, Rodríguez de Mendoza) y quizás también para el caso de Chuqui-mal (Ocumal, Luya). El mismo fenómeno, igualmente de muy baja frecuencia, se ha observado en el análisis de antropónimos (Zevallos, 1966: 4). Al mismo tiempo, la falta de existencia de palabras compuestas por elementos castellanos y chacha es una evidencia más del escaso respectivamente inexistente uso de la lengua chacha a inicios de la Conquista.

\section{Conclusiones}

\subsection{Síntesis}

En conclusión, el trabajo de campo ha podido comprobar la recurrencia de las terminaciones -mal y las variantes de -gat en una cantidad considerable de topónimos de la zona antiguamente habitada por los pueblos chachapoyas, con un centro importante en varios distritos de las actuales provincias de Luya y Chachapoyas, en el departamento de Amazonas (figura. 3), donde también se constata la más alta concentración de restos arqueológicos de la cultura en cuestión. Así mismo, las muestras tomadas en el trabajo de campo en algunos distritos de las provincias mencionadas han demostrado que, en los distritos visitados, existe una frecuencia de uso de topónimos con las terminaciones en cuestión todavía mucho más alta de lo que se ha podido extraer de las bases de datos oficiales que contienen registros de topónimos, lo que deja suponer una situación similar en los demás distritos de la zona.

La correspondencia, aunque con brechas todavía por explicar, entre el área linguiística y el ámbito delimitado por investigaciones histórico-arqueológicas, las evidencias antroponímicas y los resultados de la revisión linguística de los ámbitos vecinos de la cultura chachapoya en su conjunto permiten concluir que ambas terminaciones toponímicas formaban parte de un sistema linguiístico propio y común de los pueblos de la cultura chachapoya. Mientras en el caso de -mal, se puede asumir que se trata de un morfema propio del idioma chacha, el análisis de -gat ha demostrado que probablemente se trata del vestigio de un idioma anterior, el cual lo asumió el idioma chacha, o de un préstamo linguiístico de un idioma paralelo al chacha.

En cuanto a -gat, se ha comprobado el significado propuesto de 'recurso hídrico'. Por las diferentes variedades de recursos hídricos identificados en el trabajo de campo (pozos, quebradas, ríos), es de suponer que -gat no alude a ninguna forma de manifestación en específico. En el caso de -mal, se ha podido 
reunir evidencia que permite postular un significado de 'lugar donde abunda un determinado recurso', más que 'pampa' o 'llanura', ya que no todos los sitios identificados en el trabajo de campo estaban relacionados con pampas o llanuras.

Además de las dos terminaciones estudiadas, se ha constatado que el morfema gach- puede aparecer en su calidad de raíz en la formación de topónimos y puede ser colocado como primer -o incluso único ${ }^{17}$ - elemento de un topónimo. Como los topónimos comprobados en el trabajo de campo hacen referencia a recursos hídricos, al igual que los topónimos con alguna terminación derivada de -gat, se deduce el significado de 'agua' para el morfema -gat en cualquier posición de la palabra.

\subsection{Discusión y recomendaciones}

Los resultados del presente trabajo permiten formular una serie de puntos por investigar y recomendaciones para investigaciones futuras sobre la lengua de los antiguos chachapoyas:

1. Para sacar conclusiones más definitivas sobre la extensión del idioma chacha, hace falta identificar y comprobar más topónimos de la lengua en cuestión, empezando por la revisión de las terminaciones toponímicas ya propuestas: -lap, -huala y -lon, así como la determinación de su significado respectivo.

2. En la presente investigación se han tomado en cuenta únicamente fuentes actuales. Para tener una idea más precisa de la distribución original de los topónimos chacha, habrá que revisar fuentes del inicio de la época colonial, así como identificar factores y tendencias de sustitución de topónimos en las zonas de la cultura chachapoya donde las terminaciones toponímicas son menos frecuentes, como por ejemplo patrones de asentamiento y otros datos etnográficos.

3. Una vez que se tenga una base de datos más consolidada de posibles características y vocabulario chacha, se podrá investigar una posible filiación linguística del idioma chacha, así como la llegada del quechua a la zona, la cual todavía no ha podido ser fechada con seguridad; las palabras compuestas por morfemas quechua y chacha identificadas en el presente trabajo constituyen un primer indicio.

17 Por ejemplo, en el caso de Gache (Lámud, Luya), tomando en cuenta que no necesariamente se ha conservado la forma original y completa del topónimo en este caso. 
4. Se ha comprobado que la recurrencia de las terminaciones toponímicas investigadas en el presente trabajo es todavía mucho más alta de lo que hacen suponer las fuentes disponibles de registro de topónimos. Por lo tanto, es necesario realizar más trabajo de campo, sobre todo en las zonas que presentan escasas evidencias linguiísticas del idioma de los antiguos chachapoyas, aunque muy probablemente la frecuencia de topónimos chacha en estas áreas quedará por debajo del promedio identificado en las provincias de Chachapoyas y Luya del departamento de Amazonas.

5. Por último, si bien es importante investigar las preguntas linguiísticas más preponderantes dentro del área de la cultura chachapoya, no se ha de perder de vista las zonas alrededor de lo que se supone que era el área del idioma chacha, con la finalidad de aislar correctamente las características netamente chacha y contrastarlas con los idiomas de las zonas colindantes, tomando en cuenta el análisis de recurrencia y distribución de -gat y -mal en el presente trabajo como punto de partida.

\section{Referencias bibliográficas}

CERRÓN-PALOMINO, R. (1983). Guía para estudios de toponimia. Lima: Universidad Nacional Mayor de San Marcos.

CERRÓN-PALOMINO, R. (2008). Voces del Ande. Ensayos sobre onomástica andina. Lima: PUCP.

CERRÓN-PALOMINO, R. (2015). «Toponimia andina: problemas y métodos». En Lexis, XXXIX (1); pp. 183-197.

CHAPARRO, C. (1985). Fonología y lexicón del quechua de Chachapoyas. Lima: Editorial Sagsa.

CHÁVEZ, A. (2003). La toponimia en la zona de Áncash. Con referencia sobre el topónimo SHIQUI. Lima: Editorial Hozlo S.R.L.

ESPINOZA, V. (1967). «Los señoríos étnicos de los chachapoyas y la alianza hispanochacha» en Revista Histórica, 30; pp.224-333.

KAUFFMAN, F. y Ligabue, G. (2003). Los chachapoyas(s). Moradores ancestrales de los Andes amazónicos peruanos. Lima: UAP.

KOSCHMIEDER, K. (2012). Jucusbamba. Investigaciones arqueológicas y motivos Chachapoya en el norte de la Provincia de Luya, Departamento Amazonas, Perú. Lima: Tarea Asociación Gráfica Educativa.

LANGLOIS, L. (1939). Utcubamba, investigaciones Arqueológicas en este valle del departamento de Amazonas (Perú). Lima.

LERCHE, P. (1986). Häuptlingstum La Jalca - Bevölkerung und Ressourcen bei den Vorspanischen Chachapoya, Peru. Berlin: Dietrich Reimer Verlag. 
LERCHE, P. (1995). Los Chachapoya y los símbolos de su historia. Lima: Ediciones y Servicios César Gayoso.

RUIZ, A. (1972). La alfarería de Cuelap: Tradición y cambio (tesis). UNMSM.

SCHJELLERUP, I. (2005). Incas y españoles en la conquista de los chachapoya. Lima: PUCPIFEA.

SOLIS, G. (1998). La gente pasa los nombres quedan. Introducción en la toponimia. Lima: G. Herrera Editores.

TAYLOR, G. (1996). La tradición oral quechua de Chachapoyas. Lima: IFEA.

TAYLOR, G. (2000). Estudios lingüísticos sobre Chachapoyas. Lima: IFEA-UNMSM.

TAYLOR, G. (2006). Diccionario quechua Chachapoyas Lamas. Lima: IFEA-IEP.

TORERO (1989). «Áreas toponímicas e idiomas en la sierra norte peruana. Un trabajo de recuperación linguiística» en Revista Andina, Año 7; pp. 217-248.

TORERO, A. (2002). Idiomas de los Andes. Lingüística e Historia. Lima: IFEA.

VALQUI, J. (2003). «Rastreo a la extinta lengua de los chachapoyas» en Lengua y Sociedad, 5; pp.62-71.

ZEVALLOS Q., J. (1966). «Onomástica prehispánica de Chachapoyas». En Revista Lenguaje y Ciencias, 35; pp. 3-18. 